\title{
MicroRNA-141 inhibits proliferation of gastric cardia adenocarcinoma by targeting MACC1
}

\author{
Shubin $\mathrm{Li}^{1}$, Jixian $\mathrm{Zhu}^{2}$, Junjie $\mathrm{Li}^{1}$, Shubo $\mathrm{Li}^{3}$, Bin $\mathrm{Li}^{1}$
}

\author{
1Department of Cardiothoracic Surgery, Xinxiang Central Hospital, Xinxiang, Henan, \\ China \\ 2Department of Cardiothoracic Surgery, The First People's Hospital of Xinxiang, \\ Xinxiang, Henan, China \\ ${ }^{3}$ Xinxiang Central Blood Station, Xinxiang, Henan, China
}

Submitted: 13 December 2016

Accepted: 23 February 2017

Arch Med Sci 2018; 14, 3: 588-596

DOI: https://doi.org/10.5114/aoms.2017.68757

Copyright $\odot 2017$ Termedia \& Banach

\section{Abstract}

Introduction: Abnormal expression of microRNAs (miRNAs) contributes to cancer development through regulating proliferation, apoptosis and drug resistance in cancer cells. The present study was designed to explore the effect and mechanism of miR-141 on gastric cardia adenocarcinoma (GCA). Material and methods: Forty-one paired GCA tissues and adjacent normal tissues were obtained from GCA patients never treated by chemotherapy or radiotherapy. QRT-PCR was used to detect the expression level of miR-141 in GCA. Effects of miR-141 on cell proliferation and apoptosis were detected in vitro. Western blot analysis was used to determine the downstream targets of miR-141.

Results: In the present study, our data showed that miR-141 was significantly decreased and correlated with advanced TNM stage and lymph node metastases in GCA. In addition, we found that miR-141 could inhibit cell proliferation and induce cell apoptosis in AGS cells. Moreover, MACC1 was predicted as a possible target of miR-141. The luciferase reporter assay proved that miR-141 could suppress MACC1 directly by binding to its 3'-UTR. Further studies showed that miR-141 influenced the MEK/ERK and p38 MAPK signaling pathways.

Conclusions: Our findings demonstrated that miR-141 expression was associated with GCA progression. MACC1, working as one possible target of miR141 , may contribute to the process. MiR-141 is expected to be a potential therapeutic target for the treatment of GCA patients.

Key words: miR-141, MACC1, gastric cardia adenocarcinoma, MEK/ERK, p38 MAPK.

\section{Introduction}

Gastric cancer is one of the most frequent cancers in the world, especially in less developed regions [1]. Gastric cardia adenocarcinoma (GCA) is an adenocarcinoma that originates from the epithelial cells located in the connection between the stomach and esophagus and is among the most common types of gastric cancer [2]. Incidence of GCA in Asia has been increasing in recent years. Currently, surgery is a curative treatment for GCA patients [3]. There has been no effective post-surgery treatment for GCA. Metastasis and post-surgery recurrence rates in GCA are as high as $40-65 \%$ [4]. Thus, accurate and predictive markers and new therapeutic methods are urgently needed.

\author{
Corresponding author: \\ Shubin Li \\ Department of \\ Cardiothoracic Surgery \\ Xinxiang Central Hospital \\ Xinxiang 453000 \\ Henan, China \\ E-mail: shubinli0921@126. \\ com
}


MicroRNAs (miRNAs) are a class of endogenous small non-coding RNAs which suppress their target mRNAs expression by binding to the $3^{\prime}$ untranslated region (3'-UTR) based on sequence complementarity as well as accessibility of the potential target site. MiRNAs negatively regulate gene expression by either translational inhibition or degradation of target mRNAs [5]. Increasing evidence has shown that the abnormal expression of miRNAs plays important roles in many biological processes, including cell proliferation, invasion, apoptosis, and metastasis $[6,7]$. In recent years, numerous miRNAs have been shown to be involved in cancer progression. For example, Wang et al. showed that miR-124 could act as a tumor suppressor inhibiting cell proliferation and invasion by targeting $\mathrm{B} 7-\mathrm{H} 3$ in osteosarcoma [8]. Li et al. suggested that downregulated miR-506 expression facilitated pancreatic cancer progression and chemoresistance via SPHK1/Akt/NF- $\kappa B$ signaling [9]. Zhang et al. showed that miR-152 regulated metastases of non-small cell lung cancer cells by targeting neuropilin-1 [10]. However, the function and mechanism of miR-141 in GCA have not been systematically studied.

In the present study, we aimed to determine the expression of miR-141 in GCA, analyze the association between miR-141 expression and tumor progression, and further to explore the possible underlying mechanism.

\section{Material and methods}

\section{Tissue samples}

A total of 41 patients with GCA who underwent curative surgery at the Department of Cardiothoracic Surgery, Xinxiang Central Hospital between 2013 and 2014 were included in this study. Fresh samples from pathologically representative tumor regions and paired adjacent normal gastric mucosal tissues were obtained. These tissues were stored at $-80^{\circ} \mathrm{C}$ until the proteins and RNA were extracted. The pathological nature of each specimen was confirmed by examination with hematoxylin and eosin staining. Prior treatments, such as radiotherapy or chemotherapy, were not used before surgery in any of the cases. This study was approved by the ethics committee of Xinxiang Central Hospital. Informed consent was obtained from all patients.

\section{RNA isolation and quantitative real-time polymerase chain reaction}

Total RNA was extracted using Trizol (Invitrogen) according to the manufacturer's instructions. Two micrograms of total RNA from each sample was reverse transcribed into complementary DNA (cDNA) using the RNA PCR Kit (Takara). Re- al-time quantitative PCR was performed on the ABI PRISM 7500 Sequence Detector (Applied Biosystems). Then qRT-PCR was performed to quantify the expression level of miR-141 with SYBR Green PCR Master Mix (Applied Biosyste$\mathrm{ms}$ ) according to the manufacturer's instructions. MiR-141 expression normalized to U6 was calculated using the comparative $\mathrm{Ct}$ method formula: $2^{-\Delta \Delta C t}$. The primer sequences were as follows: miR141 forward, 5'-AAGGAGAGAGCGGGAAGCTA-3', and reverse, 5'-TTGTTGCAACTGCCTCTGGA-3'; U6 forward, 5'-GTTGGAGGTCGGAGTCAACGGA-3', and reverse, 5'-GAGGGATCTCGCTCCTGGAGGA-3'.

\section{Cell lines and cell culture}

The human gastric cancer cell line AGS was purchased from the American Type Culture Collection (ATCC) and cultured in DMEM medium (Gibco) supplemented with $10 \%$ fetal bovine serum (FBS, Gibco) and $100 \mathrm{mg} / \mathrm{ml}$ penicillin-streptomycin. The cells were maintained under a humidified atmosphere of $5 \% \mathrm{CO}_{2}$ at $37^{\circ} \mathrm{C}$. The miR-141 mimics, NC mimics, miR-141 inhibitor, and NC inhibitor, synthesized and purified by GenePharma Company (Shanghai), were transfected into AGS cells at a final concentration of $50 \mathrm{nM}$ using X-treme in serum-free Opti-MEM (Invitrogen). Transfection efficiency was confirmed by qRT- PCR.

\section{Cell proliferation assay}

The cell proliferation assay was performed by 3-(4,5-dimethylthiazol-2-yl)-2 5-diphenyltetrazolium bromide (MTT) assay. After transfection with the indicated mimics or inhibitor, cells were plated in 96-well plates at $5 \times 10^{3}$ per well in $200 \mu \mathrm{l} \mathrm{cul-}$ ture medium. After $24 \mathrm{~h}, 48 \mathrm{~h}$ and $72 \mathrm{~h}, 20 \mu \mathrm{l}$ of $5 \mathrm{mg} / \mathrm{ml}$ MTT was added to each well, then the cells were incubated for $4 \mathrm{~h}$ before $150 \mu \mathrm{l}$ of DMSO was added. Once the insoluble crystals were completely dissolved, the absorbance values at $570 \mathrm{~nm}$ were measured by a micro-enzyme-linked immunosorbent assay plate reader. All results were obtained from at least three experiments with triplicate reactions.

\section{Cell apoptosis analysis}

Cell apoptosis analysis was performed with the Annexin-V FITC Apoptosis Detection Kit (Invitrogen) according to the manufacturer's instructions. The cells were seeded into 12 -well plates at a density of $5 \times 10^{5}$ cells per well in triplicate and transfected with the indicated mimics or inhibitor for $48 \mathrm{~h}$, and then examined using a flow cytometer, and the apoptosis populations were determined by ModFit software. All results were obtained from at least three experiments with triplicate reactions. 


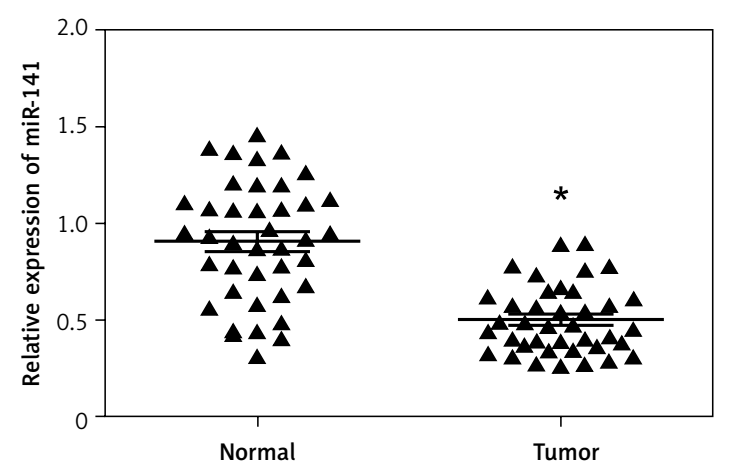

Figure 1. MiR-141 was decreased in GCA tissues compared to adjacent normal tissues. Relative expression level of miR-141 in GCA tissues and adjacent normal tissues was detected by qRT-PCR ${ }^{\star} P<0.05$.

\section{Luciferase reporter assay}

To evaluate the effect of miR-141 on MACC1 3'-UTR, firefly and Renilla luciferase activity were measured with the Dual-Luciferase Reporter Assay System (Promega). The psi-CHECK2 or psiCHECK2 vectors containing MACC1 3'-UTR-Wt or MACC1 3'-UTR-Mut were cotransfected with miR$141 \mathrm{mimics} / \mathrm{NC}$ mimics into cells using Oligofectamine (Invitrogen).

Table I. Correlation between miR-141 expression and clinicopathologic features

\begin{tabular}{|c|c|c|c|c|}
\hline Variable & \multicolumn{4}{|c|}{ expression expression } \\
\hline \multicolumn{5}{|l|}{ Age [years]: } \\
\hline$<50$ & 17 & 11 & 6 & 0.680 \\
\hline$\geq 50$ & 24 & 14 & 10 & \\
\hline \multicolumn{5}{|l|}{ Gender: } \\
\hline Male & 19 & 11 & 8 & 0.707 \\
\hline Female & 22 & 14 & 8 & \\
\hline \multicolumn{5}{|c|}{ Depth of invasion: } \\
\hline $\mathrm{T} 1 / 2$ & 26 & 18 & 8 & 0.154 \\
\hline $\mathrm{T} 3 / 4$ & 15 & 7 & 8 & \\
\hline \multicolumn{5}{|c|}{ History of UGIC: } \\
\hline Negative & 23 & 16 & 7 & 0.202 \\
\hline Positive & 18 & 9 & 9 & \\
\hline \multicolumn{5}{|l|}{ TNM stage: } \\
\hline I, II & 21 & 9 & 12 & 0.015 \\
\hline III, IV & 20 & 16 & 4 & \\
\hline \multicolumn{5}{|c|}{ Lymph node metastases: } \\
\hline Negative & 26 & 12 & 14 & 0.010 \\
\hline Positive & 15 & 13 & 2 & \\
\hline
\end{tabular}

\section{Western blot analysis}

The proteins were extracted from the transfected cells. Briefly, the cells were washed with PBS and suspended in a lysis buffer. The lysates were collected and stored at $-20^{\circ} \mathrm{C}$. Supernatant protein concentration was determined using the Bio-Rad protein assay system (Bio-Rad). Equal amounts of protein samples were fractionated by SDS-PAGE and transferred to a nitrocellulose membrane. The blots were blocked for $2 \mathrm{~h}$ with $5 \%$ nonfat milk at room temperature, then immunoblotted with primary antibodies in PBS and incubated at $4^{\circ} \mathrm{C}$ overnight. The membranes were washed with PBS-T and then incubated with secondary antibodies. The blots were scanned and the band density was measured using the Quantity One imaging software. The primary antibodies anti-Bax, anticaspase-3, anti-Bcl-2, anti-MACC1 and anti-GAP$\mathrm{DH}$ were purchased from Santa Cruz Biotechnology. The primary antibodies of anti-p-p38, anti-pMEK, anti-p-ERK, anti-P38, anti-MEK and anti-ERK were purchased from Cell Signaling Technology.

\section{Statistical analysis}

Statistical analyses were performed using SPSS 18.0 software and GraphPad Prism 5 . All data are expressed as mean \pm SD. Statistical evaluation of the data was performed with one-way ANOVA or Student's $t$-test or the $\chi^{2}$ test. A $p$-value less than 0.05 was considered statistically significant.

\section{Results}

\section{MiR-141 was downregulated in gastric cardia adenocarcinoma tissues}

To validate the function of miR-141 in gastric cardia adenocarcinoma (GCA), we explored the expression of miR-141 in 41 pairs of GCA tissue and matched adjacent normal tissues by using qRT-PCR. Our results revealed that the expression level of miR-141 in tumor tissues was generally lower than the matched adjacent normal tissues (Figure 1, $p<0.05)$. To explore the correlation between miR-141 expression and pathological characteristics in GCA tumors, we divided the 41 GCA patients into a high expression group and a low expression group according to the median expression of miR-141 in all GCA samples. Our data showed that low miR-141 expression was correlated with TNM stage and lymph node metastases (Table I; $p<0.05$ ). There was no significant correlation between miR-141 expression and the other clinicopathological parameters, including patients' age, gender, depth of invasion, and family history of upper gastrointestinal tract cancer (UGIC). Thus, these observations suggested that 
miR-141 was associated with the progression and development of GCA.

\section{MiR-141 suppressed cell proliferation}

To examine the influence of miR-141 on GCA progression and the possible mechanism, we transfected GCA cells with miR-141 mimics or miR-141 inhibitor. The transfection efficiency was validated by qRT-PCR (Figure $2 \mathrm{~A}, p<0.05$ ). The MTT assay was used to evaluate cell viability directly. We found that miR-141 mimics significantly reduced GCA cell viability compared to the control group, while the inhibition of miR-141 promoted cell viability (Figure $2 \mathrm{~B}, p<0.05$ ). To investigate the potential mechanisms underlying cell proliferation, flow cytometry was used. Apoptosis analysis showed that miR-141 mimics promoted cell apoptosis compared to the control group, while the inhibition of miR-141 inhibited cell apoptosis compared with the control group (Figure $2 \mathrm{C}$, $p<0.05)$. Furthermore, we explored the expression level of apoptosis-related proteins (Bax, Bcl-2 and caspase-3). Western blot showed that miR141 mimics increased the expression of Bax and caspase-3 protein. Contrarily, Bcl-2 expression was depressed, while the inhibition of miR-141 decreased the expression of Bax and caspase- 3 protein and increased the expression of $\mathrm{Bcl}-2$ (Figure $2 \mathrm{D}, p<0.05)$. These findings suggested that miR-141 inhibited GCA cell proliferation by promoting cell apoptosis.

\section{MACC1 was a direct target of miR-141}

To explore the mechanism underlying the growth inhibition by miR-141 in GCA cells, we used publicly available algorithms (TargetScan, miRan$\mathrm{da}$, miRwalk) to predict the potential targets of miR-141 in humans. MACC1 was predicted as a potential target of miR-141 by the three algorithms (Figure 3 A). Through Western blot, we found that MACC1 was significantly decreased after transfection of miR-141 mimics and increased after transfection of miR-141 inhibitor (Figure 3 B, $p<0.05$ ). The dual luciferase reporter assay was employed to confirm whether miR-141 could directly bind to $3^{\prime}$-UTR of MACC1. We found that miR-141 upregulation significantly suppressed the relative luciferase activity of the wild type (Wt) reporter plasmid, but that of the mutant (Mut) reporter plasmid was unaffected (Figure $3 C, p<0.05$ ). These results suggested that miR-141 directly recognized the $3^{\prime}$-UTR of MACC1 mRNA and inhibited MACC1 translation.

\section{MiR-141 inhibited MEK/ERK and activated p38 MAPK signaling}

To further explore the possible mechanism, we examined the MEK/ERK and p38 MAPK sig- naling pathway. We found that miR-141 mimics significantly activated the p38 signaling pathway. Meanwhile, transfection of miR-141 mimics inhibited activation of MEK. The expression of $p$-ERK was decreased but there was no change in the total-ERK as compared with NC mimics. The inhibition of miR-141 inhibited the p38 signaling pathway. Meanwhile, transfection of miR-141 inhibitor promoted activation of MEK. The expression of p-ERK was increased but there was no change in the total ERK as compared with NC inhibitor (Figure 4, $p<0.05$ ). Therefore, the present study indicated that miR-141 could inhibit the cell proliferation of GCA by regulation of MEK/ERK and p38 MAPK signaling pathways.

\section{Discussion}

Accumulating data showed that miR-141 was a significantly dysregulated miRNA in various cancers and was associated with cancer development. For example, Wang et al. reported that increased miR-141 expression was associated with diagnosis and favorable prognosis of patients with bladder cancer [11]. Liu et al. suggested that miR141 was increased in nasopharyngeal carcinoma and involved in BRD7-mediated cell proliferation and tumor formation through suppression of the PTEN/AKT pathway in nasopharyngeal carcinoma [12]. However, Peng et al. suggested that miR-141 could inhibit glioma cell growth and metastasis by targeting TGF- $\beta 2$ [13]. Chen et al. reported that miR-141 was downregulated in renal cell carcinoma and regulated cell proliferation and metastasis by controlling EphA2 expression [14]. Huang et al. found that miR-141 inhibited gastric cancer cell proliferation and tumor growth, and the regulatory mechanism might be associated with IGF1R [15]. However, the roles of miR-141 in GCA remain unclear.

The difference of miRNAs between cancer tissues and adjacent normal tissues indicated that these specific miRNAs were involved in the occurrence and development of cancer and could be potential targets for cancer treatment. Gastric cancer also exhibited alterations in the abundance of specific miRNAs. For example, Yin et al. suggested that miR-208a-3p suppressed cell apoptosis by targeting PDCD4 in gastric cancer [16]. Wu et al. found that miR-483-5p promoted growth, invasion and self-renewal of gastric cancer stem cells by $W n t / \beta$-catenin signaling [17]. The imbalances of substantial miRNAs in gastric cancer tissues have been proposed as potential biomarkers for prognosis [18]. In our study, we found that the expression of miR-141 was lower in GCA tissues compared to adjacent normal tissues, indicating that miR-141 may serve as a tumor suppressor in GCA progression. In addition, we found that the 

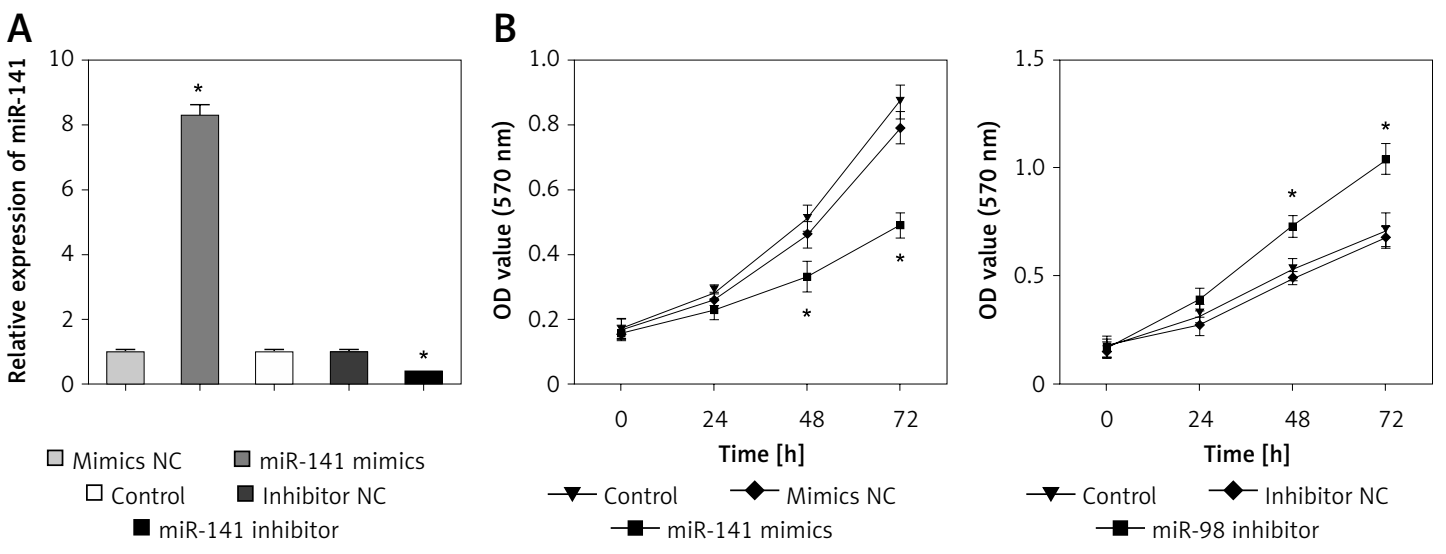

C
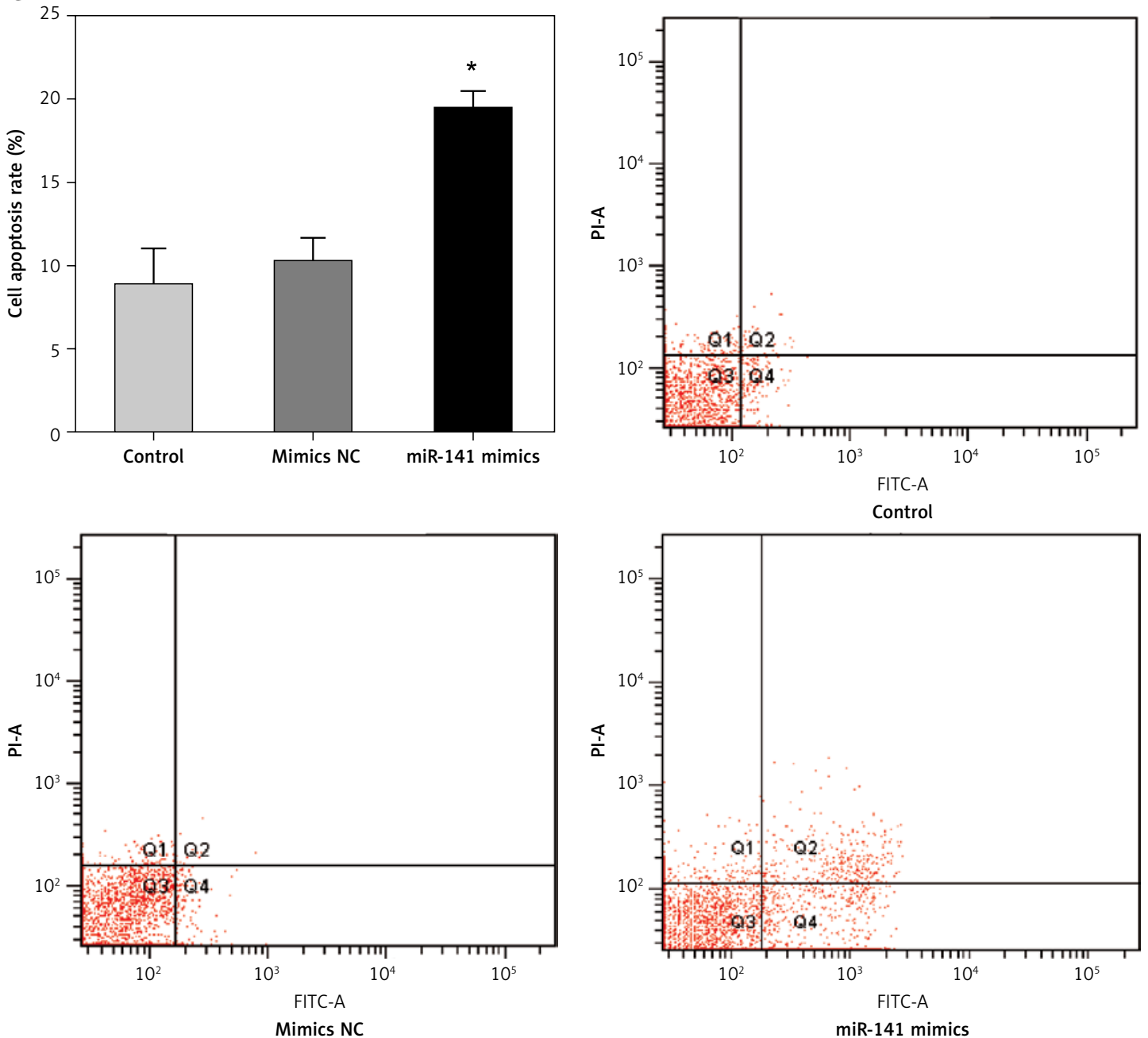

Figure 2. MiR-141 inhibited AGS cell proliferation in vitro. A - Expression of miR-141 in AGS cells transfected with miR-141 mimics, NC mimics, miR-141 inhibitor, and NC inhibitor. B - The vitality of AGS cells transfected with miR-141 mimics or miR-141 inhibitor was detected using the MTT assay. C - The apoptosis rate of AGS cells transfected with miR-141 mimics or miR-141 inhibitor was detected using the flow cytometer. D - The expression level of apoptosis-related proteins (Bax, $\mathrm{Bcl}-2$ and caspase-3) was detected by Western blot in miR-141 mimics or miR-141 inhibitor transfected AGS cells

${ }^{*} P<0.05$. 

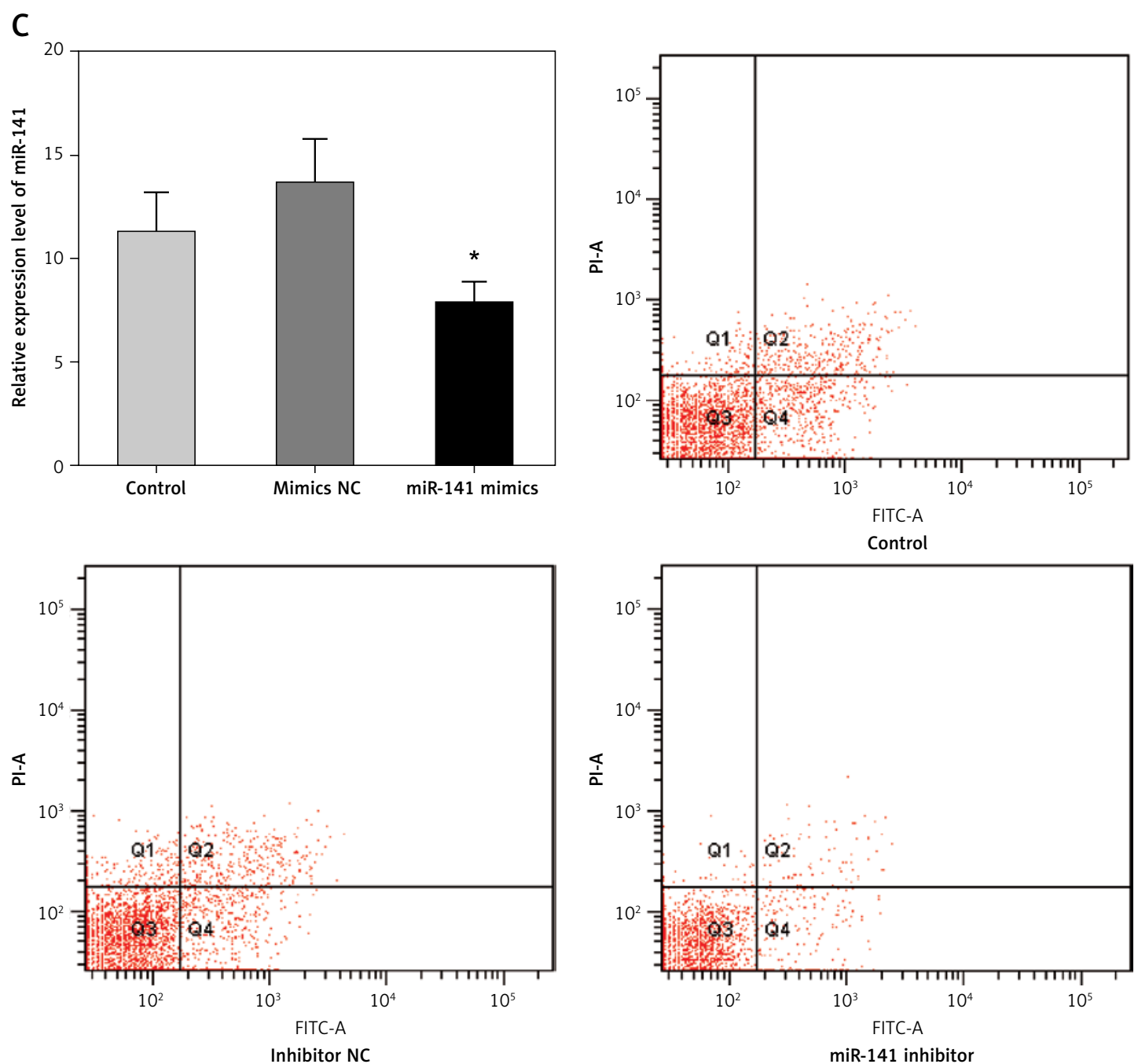

miR-141 inhibitor

D

$\mathrm{BCl}-2$
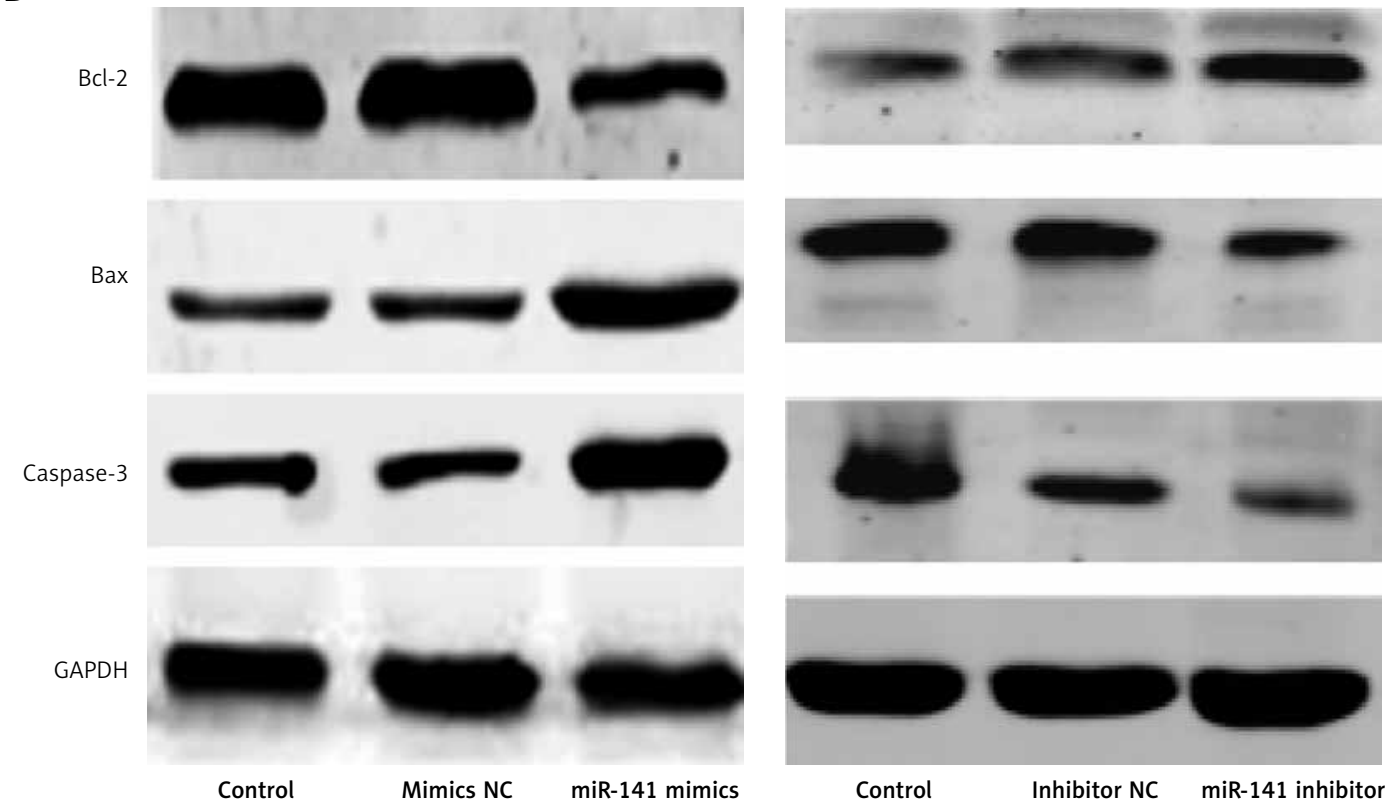

Figure 2. Cont. 
A

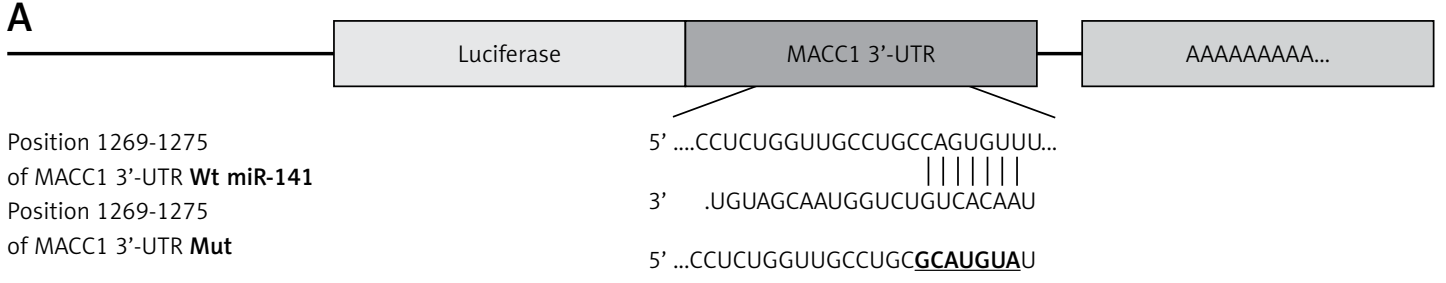

of MACC1 3'-UTR Mut 5' ...CCUCUGGUUGCCUGCGCAUGUAU

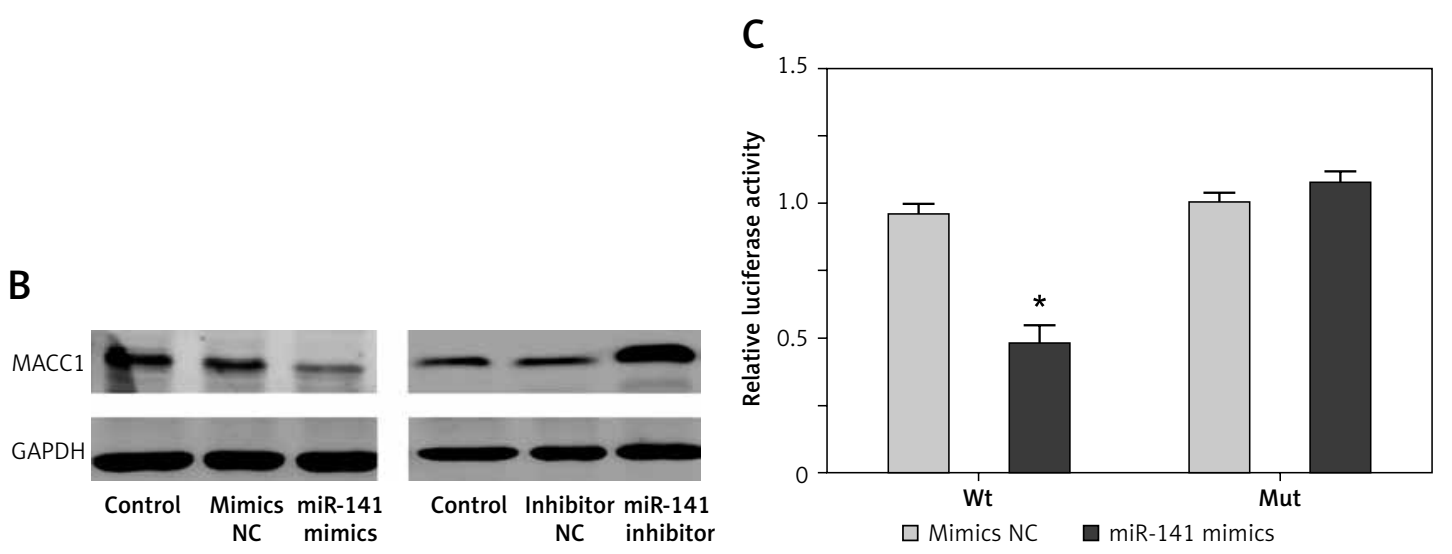

Figure 3. MACC1 was a target of miR-141 in GCA cells. A - The potential miR-141 binding sites of MACC1 3'-UTR and the Mut sequences. B - Western blot analysis of MACC1 expression in AGS cells after transfection with miR141 mimics or miR-141 inhibitor. C - AGS cells were co-transfected with miR-141 mimics or NC mimics with wild type or mutant MACC1 $3^{\prime}$-UTR. Dual luciferase reporter assay was used

${ }^{*} P<0.05$.
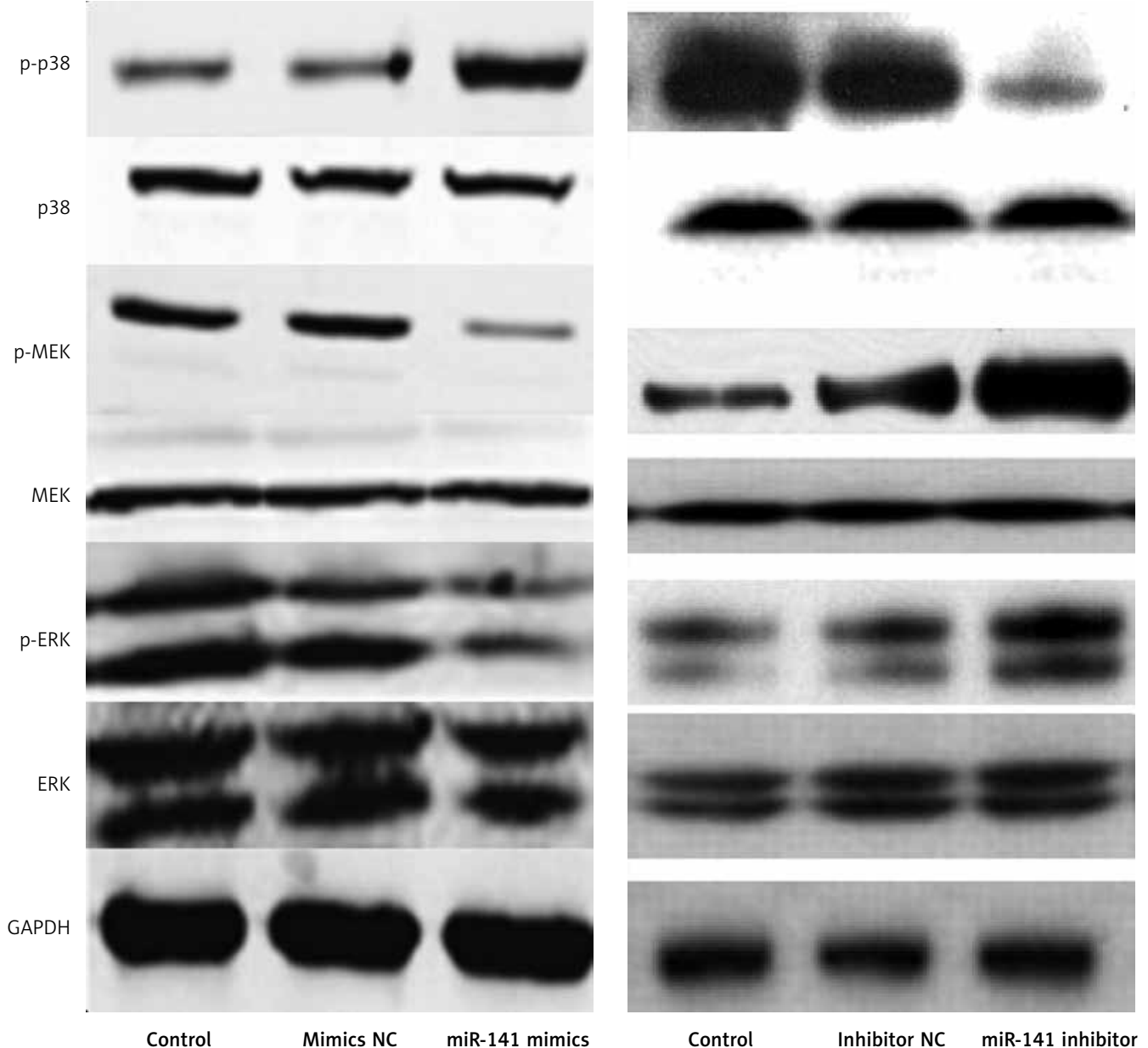

Figure 4. MiR-141 inhibited MEK/ERK and activated p38 MAPK signaling. p-p38, p38, p-MEK, MEK, p-ERK, and ERK were measured by Western blot 
low expression of miR-141 was correlated with TNM stage and lymph node metastasis. Lu et al. observed that decreased miR-141 expression in gastric cancer was significantly associated with tumor differentiation, lymph node metastasis, distant metastasis, and TNM stage, which was inconsistent with our findings [19].

To explore the possible mechanism of miR141 in GCA, we employed a gastric cancer cell model. MiR-141 mimics significantly reduced cell proliferation and increased the apoptosis of AGS cells. Further studies showed that apoptosis-related proteins were also changed. MiR-141 mimics increased protein levels of Bax and caspase-3. Conversely, Bcl-2 expression was depressed. Meanwhile, miR-141 inhibition reduced Bax and caspase- 3 protein expression and increased $\mathrm{Bcl}-2$ protein expression. The results indicated that an imbalance of Bax, caspase- 3 and $\mathrm{Bcl}-2$ might contribute to apoptosis of GCA cells, suggesting that miR-141 might serve as a tumor suppressor in GCA progression.

MiRNA is a cluster of non-coding RNA combined with specific target genes which play critical biological functions in tumor progression. To explore the possible target of miR-141, we searched three publicly available algorithms (TargetScan, miRanda, miRwalk) and found that MACC1 was a potential target of miR-141. To confirm the prediction, we introduced exogenous miR-141 into AGS cells and found that miR-141 mimics down-regulated MACC1 expression, while inhibition of miR-141 in AGS cells significantly increased MACC1 expression. The luciferase reporter assay also proved that miR-141 could suppress MACC1 directly by binding to its 3'-UTR. Previous studies have shown that MACC1 plays important roles in the development and progression of gastric cancer [20, 21]. Thus, we inferred that miR-141 might regulate GCA progression by regulating MACC1.

Metastasis-associated in colon cancer-1 (MACC1) is an oncogene first discovered in colon cancer, where its main action was thought to be promotion of metastasis [22]. The MACC1 gene is located on human chromosome 7 (7p21.1), contains seven exons and six introns, and the effective coding region of the gene consists of 2559 nucleotides [23]. Recently, a number of studies have shown that there is a close relationship between MACC1 and proliferation, metastasis and recurrence of a variety of tumors [24-26]. Zhang et al. demonstrated that MEK/ERK and p38 MAPK signaling mediated by MACC1 regulated cell proliferation, migration, and invasion in ovarian cancer [27]. In the present study, we also observed the change of MEK/ERK and p38 MAPK. We found that miR-141 mimics significantly activated the p38 signaling pathway, whereas miR-497 mimics inhibited activation of MEK and ERK. These results suggested that restoration of the expression of miR-141 suppressed MACC1, which may modulate MEK/ERK and p38 MAPK signaling in GCA.

In conclusion, our data suggested that miR-141 was downregulated in GCA tissues, and ectopic expression of miR-141 inhibited cell proliferation and induced cell apoptosis in vitro. MACC1 was identified as a target of miR-141 in GCA, and overexpression of miR-141 inhibited MEK/ERK and activated the p38 MAPK signaling pathway, indicating that miR-141 might be a potential therapeutic target for the treatment of GCA.

\section{Acknowledgments}

Shubin Li and Jixian Zhu made the same contribution to this work.

The project was supported by science and technology projects of Xinxiang City (CXGG16060).

\section{Conflict of interest}

The authors declare no conflict of interest.

\section{References}

1. Jemal A, Bray F, Center MM, Ferlay J, Ward E, Forman D. Global cancer statistics. CA Cancer J Clin 2011; 61: 6990.

2. Kelley JR, Duggan JM. Gastric cancer epidemiology and risk factors. J Clin Epidemiol 2003; 56: 1-9.

3. Leung WK, Wu MS, Kakugawa Y, et al. Screening for gastric cancer in Asia: current evidence and practice. Lancet Oncol 2008; 9: 279-87.

4. Alberts SR, Cervantes A, van de Velde CJ. Gastric cancer: epidemiology, pathology and treatment. Ann Oncol 2003; 14 Suppl. 2: ii31-36.

5. Bartel DP. MicroRNAs: target recognition and regulatory functions. Cell 2009; 136: 215-33.

6. Garzon R, Fabbri M, Cimmino A, Calin GA, Croce CM. MicroRNA expression and function in cancer. Trends Mol Med 2006; 12: 580-7.

7. Wienholds E, Plasterk RH. MicroRNA function in animal development. FEBS Lett 2005; 579: 5911-22.

8. Wang L, Kang FB, Sun N, et al. The tumor suppressor miR-124 inhibits cell proliferation and invasion by targeting B7-H3 in osteosarcoma. Tumour Biol 2016; 37 : 14939-47.

9. Li J, Wu H, Li W, et al. Downregulated miR-506 expression facilitates pancreatic cancer progression and chemoresistance via SPHK1/Akt/NF-kappaB signaling. Oncogene 2016; 35: 5501-14.

10. Zhang YJ, Liu XC, Du J, Zhang YJ. MiR-152 regulates metastases of non-small cell lung cancer cells by targeting neuropilin-1. Int J Clin Exp Pathol 2015; 8: 14235-40.

11. Wang XL, Xie HY, Zhu CD, et al. Increased miR-141 expression is associated with diagnosis and favorable prognosis of patients with bladder cancer. Tumor Biology 2015 ; 36: 877-83.

12. Liu $Y$, Zhao $R$, Wang $H$, et al. miR-141 is involved in BRD7-mediated cell proliferation and tumor formation through suppression of the PTEN/AKT pathway in nasopharyngeal carcinoma. Cell Death Dis 2016; 7: e2156. 
13. Peng T, Zhang S, Li W, Fu S, Luan Y, Zuo L. MicroRNA-141 inhibits glioma cells growth and metastasis by targeting TGF-beta2. Am J Transl Res 2016; 8: 3513-21.

14. Chen X, Wang X, Ruan A, et al. miR-141 is a key regulator of renal cell carcinoma proliferation and metastasis by controlling EphA2 expression. Clin Cancer Res 2014; 20: 2617-30

15. Huang $M$, Wu L, Qin $Y$, et al. Anti-proliferative role and prognostic implication of miR-141 in gastric cancer. Am J Transl Res 2016; 8: 3549-57.

16. Yin K, Liu M, Zhang M, et al. miR-208a-3p suppresses cell apoptosis by targeting PDCD4 in gastric cancer. Oncotarget 2016; 7: 67321-32.

17. Wu K, Ma L, Zhu J. miR-483-5p promotes growth, invasion and self-renewal of gastric cancer stem cells by Wnt/beta-catenin signaling. Mol Med Report 2016; 14: 3421-8.

18. Ueda T, Volinia S, Okumura $\mathrm{H}$, et al. Relation between microRNA expression and progression and prognosis of gastric cancer: a microRNA expression analysis. Lancet Oncol 2010; 11: 136-46.

19. Lu YB, Hu JJ, Sun WJ, Duan XH, Chen X. Prognostic value of miR-141 downregulation in gastric cancer. Genet Mol Res 2015; 14: 17305-11.

20. Shimokawa H, Uramoto H, Onitsuka T, et al. Overexpression of MACC1 mRNA in lung adenocarcinoma is associated with postoperative recurrence. J Thorac Cardiovasc Surg 2011; 141: 895-8.

21. Huang N, Wu Z, Lin L, et al. MiR-338-3p inhibits epithelial-mesenchymal transition in gastric cancer cells by targeting ZEB2 and MACC1/Met/Akt signaling. Oncotarget 2015; 6: 15222-34.

22. Jorissen RN, Gibbs P, Christie M, et al. Metastasis-associated gene expression changes predict poor outcomes in patients with Dukes stage B and C colorectal cancer. Clin Cancer Res 2009; 15: 7642-51.

23. Stein U, Smith J, Walther W, Arlt F. MACC1 controls Met: what a difference an Sp1 site makes. Cell Cycle 2009; 8: 2467-9.

24. Shimokawa H, Uramoto H, Onitsuka T, et al. Overexpression of MACC1 mRNA in lung adenocarcinoma is associated with postoperative recurrence. J Thorac Cardiovasc Surg 2011; 141: 895-8.

25. Zhang Y, Wang Z, Chen M, et al. MicroRNA-143 targets MACC1 to inhibit cell invasion and migration in colorectal cancer. Mol Cancer 2012; 11: 23.

26. Gao J, Ding F, Liu Q, Yao Y. Knockdown of MACC1 expression suppressed hepatocellular carcinoma cell migration and invasion and inhibited expression of MMP2 and MMP9. Mol Cell Biochem 2013; 376: 21-32.

27. Zhang R, Shi H, Chen Z, Wu Q, Ren F, Huang H. Effects of metastasis-associated in colon cancer 1 inhibition by small hairpin RNA on ovarian carcinoma OVCAR-3 cells. J Exp Clin Cancer Res 2011; 30: 83. 